\title{
IUE OBSERVATIONS OF STARS IN NGC 330 IN THE SMALL MAGELLANIC CLOUD
}

\author{
V. CALOI ${ }^{1}$, A. CASSATELLA ${ }^{2}$, V. CASTELLANI ${ }^{3}$, G. KLARE $^{4}$ \\ 1 Istituto Astrofisica Spaziale, Frascati, Italy \\ 2 Astr. Div., ESTEC, VILSPA, ESA, Madrid, Spain \\ 3 Physics Dept., Pisa University, Pisa, Italy \\ 4 Ladessternwarten Heidelberg-Koenigstuhl, Heidelberg, FRG
}

\begin{abstract}
Low resolution spectra from 1200 to $3300 \AA$ have been obtained, with the IUE satellite, for seven blue giants and supergiants in the young, metal-poor globular cluster NGC 330 in the Small Magellanic Cloud (SMC). A further three spectra from the IUE archive have been added to the sample, covering a range of 2.5 mag. The effective temperatures and the local reddening in the SMC have been estimated, and the star positions in the theoretical HR diagram determined, and compared with theoretical predictions for massive star evolution. Substantial discrepancies have been found.
\end{abstract}

\section{Introduction}

IUE spectra have been obtained for the young, populous, metal-poor globular cluster NGC 330 in the SMC, both by the authors (seven spectra) and from the IUE archive (three spectra). The sample covers an interval of $2.5 \mathrm{mag}$ in the visual, that is, from (probably) helium-burning to main sequence objects (cf. Carney et al. 1985). The spectra, after the usual reduction procedure, have been corrected for the effect of the time-dependent sensitivity degradation.

\section{The reddening and the effective temperatures}

For the foreground galactic reddening, a value of $0.03 \mathrm{mag}$ has been assumed (see current estimates for the nearby galactic globular cluster $47 \mathrm{Tuc}$, and the absence of the absorption feature at $2200 \AA)$.

As for the SMC, the linearity of the extinction curve does not allow a reliable simultaneous estimate of temperature and reddening. Robertson (1974) and Carney et al. (1985) have assumed low values for the total reddening, 0.02 and 0.03 respectively, on the basis of the good relation between colours and spectral types (available for a few stars).

Temperatures estimated by means of the fitting between observed IUE spectra and Kurucz models, normalized at the visual magnitude, are, on the average, consistent with colours and spectral types, if the intrinsic reddening in the SMC is assumed to be zero; (for large luminosities, the relation between colours and $\mathrm{Te}$ is generally considered independent of metal abundance.) Increasing $\mathrm{E}(\mathrm{B}-\mathrm{V})$ in the SMC up to 0.04 , we lose consistency with spectral type. We take this value as the upper limit to the local reddening. 


\section{The theoretical HR diagram}

The star luminosities have been determined on the basis of standard extinction curves, Kurucz bolometric corrections and a true distance modulus of $18.85 \mathrm{mag}$. In Figure 1 the positions of the ten cluster stars are shown (for a reddening in the SMC of 0.04), together with evolutionary tracks by Bencivenni et al. (1990, models with semiconvection and no mass loss, masses of 10, 12, 16, 20 and $25 \mathrm{M}_{\odot}$ ). Except for the two brightest and coolest supergiants, that may be associated with the final helium burning phases in a blue loop, all the other objects fall in a region where no or very few stars are expected (white region). No observed cluster member, including the fainter ones, falls on the expected main sequence.

The substantial widening of the main sequence encountered in computations including mass loss and overshooting may marginally reconcile a few objects with predictions on hydrogen burning phases, leaving the overall comparison with observations in a highly unsatisfactory situation. A similar problem has been discussed by Tuchman and Wheeler (1990), who suggest binarity as a possible solution.

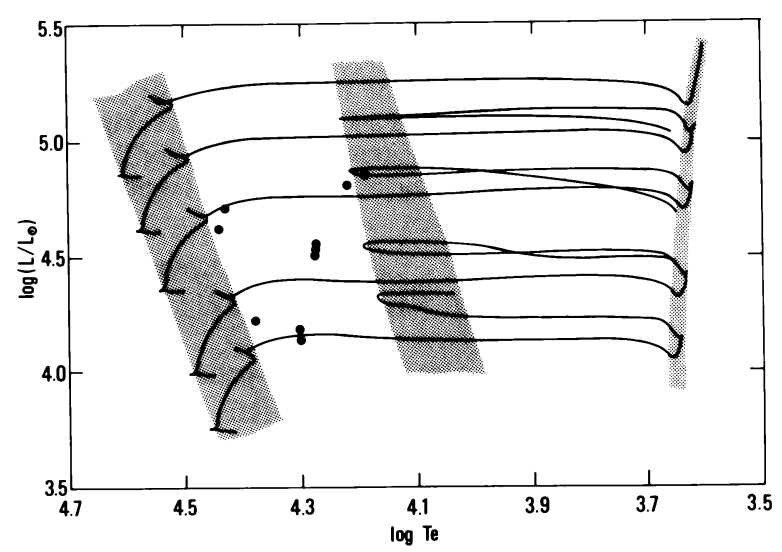

Figure 1. The positions, in the theoretical HR diagram, of ten blue giants and supergiants in NGC 330 (see text).

\section{References}

Bencivenni, D., Brocato, E., Buonanno, R. and Castellani, V. (1990), Chemical and Dynamical Evolution of Galaxies, F. Ferrini, J. Franco, F. Matteucci (eds.) (Giardini: Pisa) in press.

Carney, B.W., Janes, K.A., Flower, P.J. (1985), Astron. J. 90, 1196.

Robertson, J.W. (1974), Astron. Astroph. Suppl. 15, 261.

Tuchman, Y., Wheeler, J.C. (1990), Astroph. J. in press. 